\title{
Visible Array Waveguide Gratings for Applications of Optical Neural Probes
}

\author{
Eran Segev ${ }^{a}$, Trevor Fowler ${ }^{b}$, Andrei Faraon $^{c}$, and Michael L. Roukes ${ }^{a, b}$ \\ ${ }^{a}$ Kavli Nanoscience Institute and Departments of Physics \& Applied Physics, California \\ Institute of Technology, Pasadena, CA 91125 USA; \\ ${ }^{b}$ Division of Biology and Biological Engineering, California Institute of Technology, Pasadena, \\ CA 91125 USA; \\ ${ }^{c}$ T. J. Watson Laboratory of Applied Physics, California Institute of Technology, Pasadena, \\ CA 91125 USA
}

\begin{abstract}
In this paper we propose using Array Waveguide Gratings (AWGs), working in the visible range, in order to implement the technique of Wavelength-Division-(de)Multiplexing for multi-point stimulation of deep-brain neurons. We've developed a CMOS compatible fabrication process and fabricated two sets of AWGs, working in the red and blue wavelengths. Experimental data demonstrating the functionality of these AWGs is presented.
\end{abstract}

Keywords: WDM, AWG, Visible Photonics, Neural Probes

\section{INTRODUCTION}

In recent years, a new class of light-gated ion channels (the "opsins") have been discovered and genetically engineered to control neural modulation. ${ }^{1,2}$ Since the original discovery, the toolkit for Neural modulation using light has seen tremendous developments. ${ }^{3}$ What still lags behind, however, is an appropriate technology to target light to opsin-expressing neurons with high spatial resolution and wide coverage in three dimensions.

In the past five years several approaches have been proposed to create optical neural probes offering some degree of spatial and temporal resolution. For example, a micro machined, silicon based prototype containing multiple emission pixels was proposed in $2012 .{ }^{4}$ These probes are built as a matrix of individual silicon shanks (the linear part of the probe inserted into the brain), where each shank contains a dozen emitting pixels, each driven by an individual waveguide. The waveguides, in turn, are butt-coupled to a fiber bundle, resulting in excessive loss of light, and a rather bulky solution. A different approach, in which a tapered fiber is curved with several output windows was recently presented. ${ }^{5}$ It succeeded in demonstrating individually controlled illumination through each window. Although very appealing, there is no clear path for scaling this approach in order to create dense illumination points. An alternative approach is based on the integration of microscale Light Emitting Diodes ( $\mu$ LEDs) directly on the probe's shank,,${ }^{6,7}$ at the precise location where illumination is required. Using this approach, probes having several $\mu$ LEDs were demonstrated recently. Although this technology is promising, heat dissipation presents a very significant obstacle to the deployment of such an architecture for brain stimulation.

\section{WAVELENGTH DIVISION MULTIPLEXING}

Our alternative approach for delivering and addressing multiple illumination points deep into the brain is based on the technique of wavelength division (de)-multiplexing (WDM). WDM was originally developed in the field of optical communication in the IR, with the purpose of delivering many data channels over a single communication link. ${ }^{8}$ In this approach the spectrum of the communication link is divided into a set of spectral channels, each channel with its own specific target at the receiver side. The transmitter has the ability to split the source into

Further author information: (Send correspondence to E.S.)

E.S.: E-mail: ersegev@caltech.edu

M.L.R.: E-mail: roukes@caltech.edu

Optical Techniques in Neurosurgery, Neurophotonics, and Optogenetics II, edited by Henry Hirschberg, Steen J. Madsen,

E. Duco Jansen, Qingming Luo, Samarendra K. Mohanty, Nitish V. Thakor, Proc. of SPIE Vol. 9305,

93052L · C 2015 SPIE · CCC code: 1605-7422/15/\$18 · doi: 10.1117/12.2078599

Proc. of SPIE Vol. 9305 93052L-1 


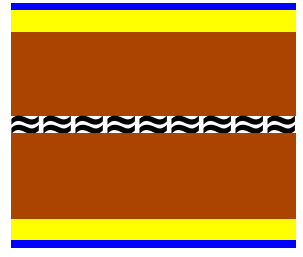

(a)

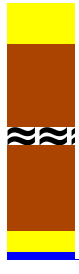

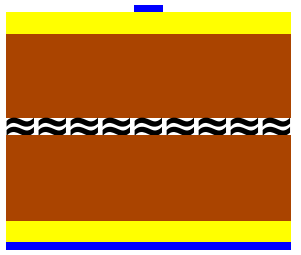

(b)
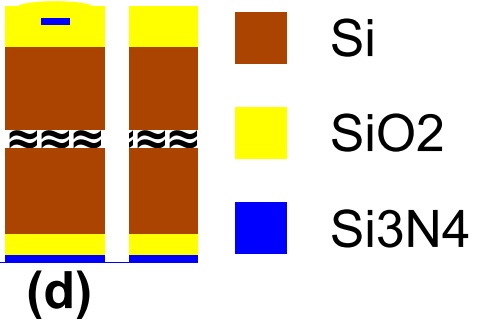

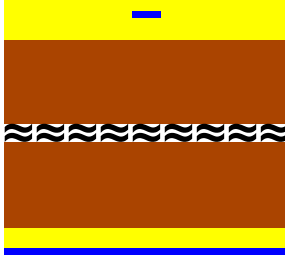

(c)

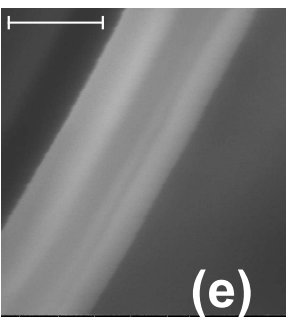

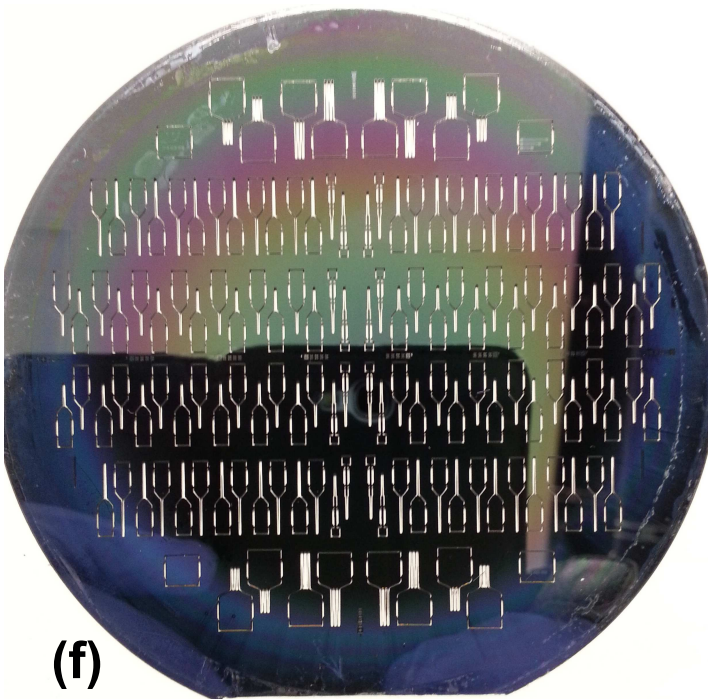

Figure 1. (a-d) Schematic drawings showing the different steps of the fabrication process. (a) The initial wafer has a layer stack comprised of the silicon substrate, a $1.5 \mu \mathrm{m}$ thick layer of thermally grown $\mathrm{SiO} 2$, and $200 \mathrm{~nm}$ of $\mathrm{Si3N} 4$. (b) Photonic waveguides and AWGs patterning. (c) Photonic circuitry covered by PECVD deposited SiO2. (d) Devices are released using a DRIE process. (e) SEM image showing a section of one of our curved waveguides, as an example of the smoothness of the waveguide fabrication process. Scale bar is $500 \mathrm{~nm}$. (f) Optical image showing an example of a completely fabricated 4" wafer, processed in Caltech's Kavli Nanoscience Institute.

specific spectral channels, individually modulate each one of them according to their data pattern, and then multiplex them back together and send them over the physical link. The receiver, in turn, has the ability to demultiplex the incoming light into its spectral components and route each channel to its target.

In this paper we report on the development of Silicon Nitride (Si3N4) based Array Waveguide Grating devices (AWGs) working at red $(673 \mathrm{~nm})$ and blue $(473 \mathrm{~nm})$ wavelengths. These AWGs can serve as the receiver building block, essential for the implementation of the WDM concept in visible wavelengths. High resolution AWGs working in the IR has been developed for almost two decades, ${ }^{9,10}$ but nevertheless, AWGs have only rarely been implemented in the visible range, ${ }^{11}$ and to the best of our knowledge have never been implemented in the blue. Si3N4 is an excellent material when targeting visible light due to its negligible absorption at that range, relatively high refractive index, and compatibility with Complementary Metal Oxide Semiconductor (CMOS) fabrication process. In addition, it is considered relatively highly biocompatible, ${ }^{12}$ which is an important consideration when targeting implantable applications.

\section{FABRICATION PROCESS}

We have developed a fully compatible CMOS fabrication process for Si3N4 based photonic circuits, which is illustrated in Fig. 1. The fabrication process starts with a 4" silicon wafers on which we thermally grow $1.5 \mu \mathrm{m}$ of Silicon Oxide $(\mathrm{SiO} 2)$ at $1000^{\circ} \mathrm{C}$. This layer is required for separating and isolating the Si3N4 waveguides from the absorptive Silicon wafer. Following, we deposit $200 \mathrm{~nm}$ of stoichiometric Si3N4 using low pressure chemical vapor deposition process (Rogue Valley Microdevices INC). This thickness was chosen so we would be able to construct the AWG using single mode, polarization insensitive waveguides, having a cross-section of $200 \mathrm{~nm} \times 200 \mathrm{~nm}$, and to insure compact devices.

Electron-beam (e-beam) writing process is used for patterning the photonic circuits. First the wafer is thoroughly cleaned in Nano-Strip for 5 minutes followed by a quick dip in buffered Hydrofluoric acid. Next, it is dehydrated on a hot-plate $\left(145^{\circ} \mathrm{C}\right)$ for 5 minutes, and then kept in Bis-trimethylsilyl-amine (HMDS) vapor environment for at least 10 minutes. Only then it is spin-coated with Ma-N 2403 (MicroChem Corp) e-beam resist, followed by a post-bake of 1 minute at $90^{\circ} \mathrm{C}$. This thorough preparation process is necessary in order to 
insure proper adhesion of the Ma-N 2403 e-beam resist to the Si3N4 layer. A Vistec EBPG-5000+, a 100kV e-beam lithography tool, is used to write the photonic patterns at a dose of $250 \mu \mathrm{C} / \mathrm{cm}^{2}$. We found that adding a resist re-flow step after the e-beam development step significantly improves the smoothness of the photonic waveguides. Samples are kept in a $145^{\circ} \mathrm{C}$ oven for 5 minutes, and then are cooled down at room temperature. This step also improves resist durability in the following etch process. A SEM image showing an example of the waveguide smoothness is shown in Fig 1(e).

The pattern is transferred to the Si3N4 layer using Inductively Coupled Plasma (ICP) etch process (Fig. 1(b)). We use pseudo-Bosch etch recipe composed of low power ICP in a composition of C4F8 and SF6 atmosphere. The power and atmosphere composition is set to insure a low etch rate of about $15 \mathrm{~nm} / \mathrm{min}$, in order to increase waveguide smoothness. After stripping the residual e-beam resist, we clad the photonic circuits by depositing $1 \mu m$ of SiO2 using a Plasma Enhanced Chemical Vapor Deposition (PECVD) process.

The last step of the fabrication process involves releasing the individual devices from the 4" wafer. Although dicing the wafer or even just scribing and breaking it would work for test devices, these processes are not compatible with the fabrication of complex geometries required for neural probe. For this reason we have developed a backside DRIE etch process. This process starts by depositing $300 \mathrm{~nm}$ of Aluminum Oxide (AlO) on the back side of the wafer. Next we use a photolithography process based on AZ 5214 photo-resist (MicroChem Corp) to pattern the boundaries of individual devices. The development of the resist using AZ 400k developer (MicroChem Corp) also etches away the AlO, thus completely transfers the pattern from the photo-resist to the AlO hard mask. The etch sequence begins with a pseudo-Bosch process, which etches through the Si3N4 and $\mathrm{SiO} 2$ layers on the backside of the Silicon wafer. Subsequently a DRIE process is used to etch trenches tranches in the wafer. The topside $\mathrm{SiO} 2$ serves as a stop layer for this process. This long etch completely etches away the photoresist mask, leaving only the $\mathrm{AlO}$ mask. This mask can be removed by dipping the wafer in $\mathrm{AZ}$ 400K developer, if desired. Figure 1(f) shows an optical image of a fully fabricated 4" wafer. Devices have been patterned in the shape of neural probes as a preparation for future research. This example wafer contains 114 probe shaped devices. Our mask design currently supports up to 20 different layouts with a total number for probes reaching 200 if desired.

\section{AWG DESIGN FABRICATION AND MEASUREMENTS}

Table 1. Summary of main AWG design parameters.

\begin{tabular}{|l|l|l|}
\hline Parameter & Device A & Device B \\
\hline Central wavelength & $673 \mathrm{~nm}$ & $473 \mathrm{~nm}$ \\
\hline Number of output channels & 7 & 9 \\
\hline Channel bandwidth & $1.07 \mathrm{~nm}$ & $0.97 \mathrm{~nm}$ \\
\hline Free Spectral Range & $18.4 \mathrm{~nm}$ & $40.5 \mathrm{~nm}$ \\
\hline PHASAR order & 31 & 9 \\
\hline Number of array waveguides & 43 & 125 \\
\hline Waveguide length difference & $11.5 \mu \mathrm{m}$ & $2.35 \mu \mathrm{m}$ \\
\hline Minimum and maximum length of array waveguides & $49 \mu \mathrm{m}, 533 \mu \mathrm{m}$ & $17.7 \mathrm{~nm}, 309 \mathrm{~nm}$ \\
\hline Footprint & $181 \times 238 \mu \mathrm{m}^{2}$ & $114 \times 156 \mu \mathrm{m}^{2}$ \\
\hline
\end{tabular}

As a proof of concept we have designed and fabricated two sets of AWG devices. Our initial designs (Device A) had a center wavelength of $673 \mathrm{~nm}$, and featured one input and seven output channels. This center wavelength was chosen given the availability of tunable laser sources in this range, and the full compatibility of the design and fabrication process with the next step of adjusting the AWGs to the blue. This step mainly involved scaling down the dimensions of the AWG, and fine adjustment of the fabrication process. An AWG design (Device B), centered around $473 \mathrm{~nm}$ is shown in Fig $2(\mathrm{a}, \mathrm{b}, \mathrm{d})$, and has one input and nine output channels. Coupling of the 

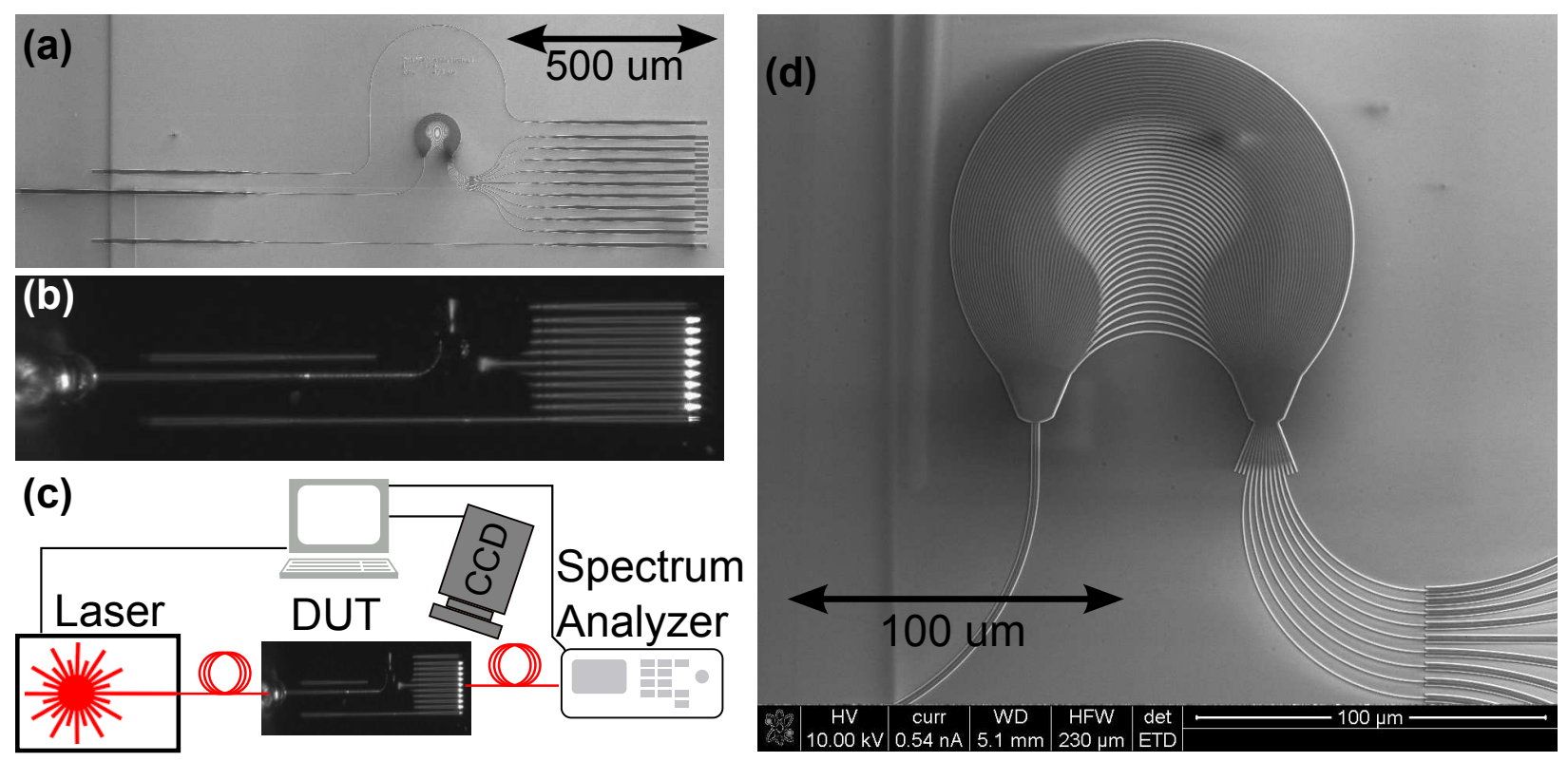

Figure 2. (a) SEM image of an AWG test chip. The image shows an AWG of type B, having 1 input channel on the left and 9 output channels on the right. Above and below the AWG there are two bypass channels, used to calibrate the AWG. (b) Dark field optical image of the AWG, obtained while the AWG is excited with broadband white light, thus all its output channels illuminate simultaneously. (c) Schematic diagram of the optical setup used to measure the AWG. (d) SEM image of one of the AWG.

AWG to the measurement setup is done by terminating its input and output ports with grating couplers. The design steps have followed the detailed theoretical derivation described in Refs. ${ }^{9,10}$ and the main parameters are listed in table 1. The footprints of these AWGs are on the order of $0.02 \mathrm{~mm}^{2}$, well suited for integration into compact neural probe designs.

Our setup for measuring the AWG is shown in Fig. 2(c). The input port of the AWG is connected to a laser. Device A is connected to a high resolution tunable red laser (Newport), whereas device B is driven by a supercontinuum laser (WhiteLase micro, Fianium). The output channels of design A are visualized by a ChargeCoupled Device (CCD) camera, which continuously monitor the channels while the wavelength of the input laser is swept. The transmitted power through each channel is calculated by integrating the image gray-level values over the effective area of the corresponding grating coupler of that channel. The drawback of this method is its rather low dynamic range and high levels of background noise. The output channels of design B are coupled to an optical fiber, and the power is measured by a spectrometer.

Subplots $(a)$ and $(b)$ of Fig. 3 show measurements of the output optical power spectrum of devices A and B, respectively, as a function of the wavelength. Each graph contains several curves, plotted using distinct colors, corresponding to different output channels of that device. Device B demonstrates channel separation levels of $3-6 \mathrm{~dB}$. The noise floor measured with this device, however, is the noise floor of the spectrometer, rather than of the AWG. Thus the signal-to-noise ratio and channel separation can be improved by increasing the input power to the AWG. Future experiments would address this issue.

\section{CONCLUSIONS}

We have functionally demonstrated AWGs working in the red and blue wavelengths. Such devices can be used as building blocks for implementing the WDM technique in the visible range. The fabrication process of these AWGs is fully compatible with CMOS-photonics fabrication processes. These AWGs have small foot-print, thus can be easily integrated into silicon based optical probes in future research. 

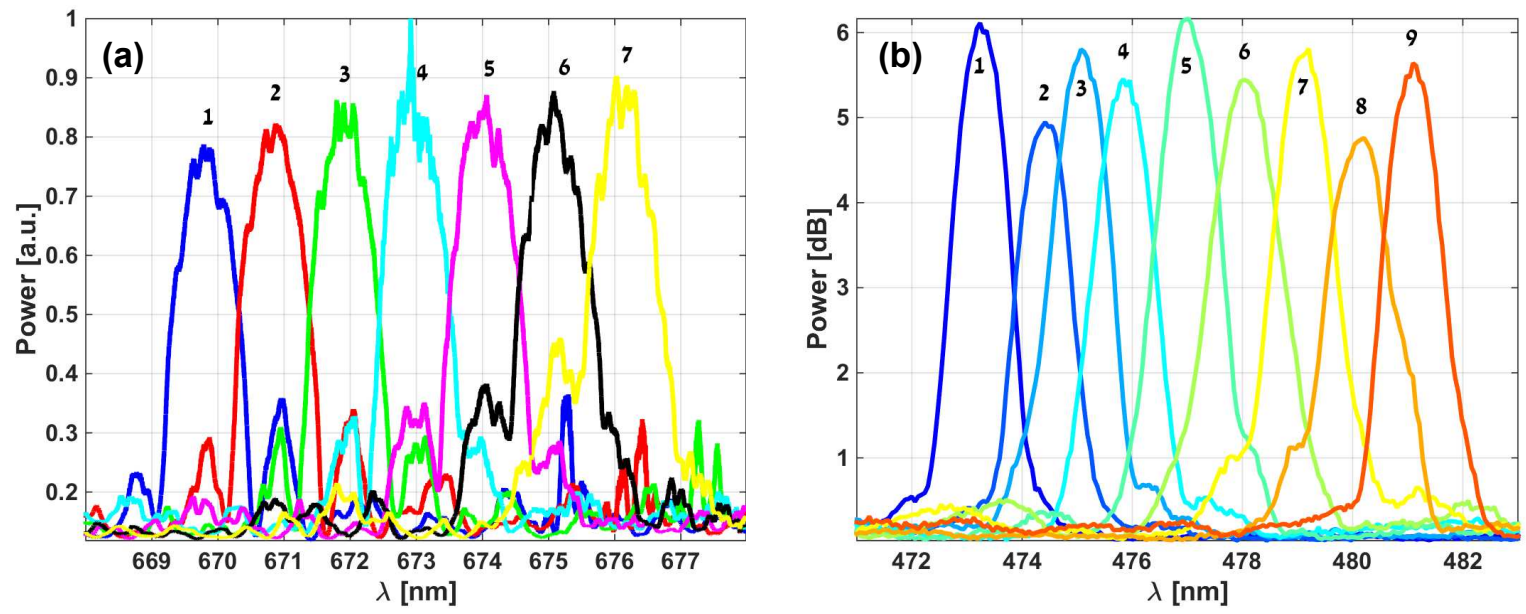

Figure 3. AWG output power spectrum as a function of the input laser wavelength. Channel numbers are marked below or above each channels' peak value. The data in (a) and (b), was obtained with devices A and B respectively.

E.S. is supported by the Kavli postdoctoral fellowship in nanoscience. This work was supported by NSF award number 1265055 and DARPA award number W911NF-14-1-0006.

\section{REFERENCES}

1. G. Nagel, T. Szellas, W. Huhn, S. Kateriya, N. Adeishvili, P. Berthold, D. Ollig, P. Hegemann, and E. Bamberg, "Channelrhodopsin-2, a directly light-gated cation-selective membrane channel," Proceedings of the National Academy of Sciences 100(24), pp. 13940-13945, 2003.

2. E. S. Boyden, F. Zhang, E. Bamberg, G. Nagel, and K. Deisseroth, "Millisecond-timescale, genetically targeted optical control of neural activity," Nature neuroscience 8(9), pp. 1263-1268, 2005.

3. J. Mattis, K. M. Tye, E. A. Ferenczi, C. Ramakrishnan, D. J. O’Shea, R. Prakash, L. A. Gunaydin, M. Hyun, L. E. Fenno, and V. Gradinaru, "Principles for applying optogenetic tools derived from direct comparative analysis of microbial opsins," Nature methods 9(2), pp. 159-172, 2011.

4. A. N. Zorzos, J. Scholvin, E. S. Boyden, and C. G. Fonstad, "Three-dimensional multiwaveguide probe array for light delivery to distributed brain circuits," Optics letters 37(23), pp. 4841-4843, 2012.

5. F. Pisanello, L. Sileo, I. A. Oldenburg, M. Pisanello, L. Martiradonna, J. A. Assad, B. L. Sabatini, and M. De Vittorio, "Multipoint-emitting optical fibers for spatially addressable in vivo optogenetics," Neuron , 2014.

6. T.-i. Kim, J. G. McCall, Y. H. Jung, X. Huang, E. R. Siuda, Y. Li, J. Song, Y. M. Song, H. A. Pao, and R.-H. Kim, "Injectable, cellular-scale optoelectronics with applications for wireless optogenetics," Science 340(6129), pp. 211-216, 2013.

7. E. Stark, T. Koos, and G. Buzski, "Diode probes for spatiotemporal optical control of multiple neurons in freely moving animals," Journal of Neurophysiology 108(1), pp. 349-363, 2012.

8. C. A. Brackett, "Dense wavelength division multiplexing networks: Principles and applications," Selected Areas in Communications, IEEE Journal on 8(6), pp. 948-964, 1990.

9. M. K. Smit and C. Van Dam, "Phasar-based wdm-devices: Principles, design and applications," IEEE Journal of Selected Topics in Quantum Electronics 2(2), pp. 236-250, 1996.

10. K. Okamoto, Fundamentals of optical waveguides, Academic press, 2010.

11. Y. Komai, H. Nagano, K. Okamoto, and K. Kodate, "Compact spectroscopic sensor using a visible arrayed waveguide grating," Japanese journal of applied physics 45(8S), p. 6742, 2006.

12. M. Mazzocchi and A. Bellosi, "On the possibility of silicon nitride as a ceramic for structural orthopaedic implants. part i: processing, microstructure, mechanical properties, cytotoxicity," Journal of Materials Science: Materials in Medicine 19(8), pp. 2881-2887, 2008. 